\title{
Can the clustered dark matter and the smooth dark energy arise from the same scalar field ?
}

\author{
T. Padmanabhan f and T. Roy Choudhury \\ IUCAA, Ganeshkhind, Pune, India 411007
}

(Dated: October 29, 2018)

\begin{abstract}
Cosmological observations suggest the existence of two different kinds of energy densities dominating at small $(\lesssim 500 \mathrm{Mpc})$ and large $(\gtrsim 1000 \mathrm{Mpc})$ scales. The dark matter component, which dominates at small scales, contributes $\Omega_{m} \approx 0.35$ and has an equation of state $p=0$, while the dark energy component, which dominates at large scales, contributes $\Omega_{V} \approx 0.65$ and has an equation of state $p \simeq-\rho$. It is usual to postulate weakly interacting massive particles (WIMPs) for the first component and some form of scalar field or cosmological constant for the second component. We explore the possibility of a scalar field with a Lagrangian $L=-V(\phi) \sqrt{1-\partial^{i} \phi \partial_{i} \phi}$ acting as both clustered dark matter and smoother dark energy and having a scale-dependent equation of state. This model predicts a relation between the ratio $r=\rho_{V} / \rho_{\text {DM }}$ of the energy densities of the two dark components and expansion rate $n$ of the universe [with $a(t) \propto t^{n}$ ] in the form $n=(2 / 3)(1+r)$. For $r \approx 2$, we get $n \approx 2$ which is consistent with observations.
\end{abstract}

The most conservative explanation of the current cosmological observations will require two components of dark matter. (a) First one is a dust component with the equation of state $p=0$ contributing $\Omega_{m} \approx 0.35$. This component clusters gravitationally at small scales $(l \lesssim 500 \mathrm{Mpc}$, say) and will be able to explain observations from galactic to supercluster scales. (b) The second one is a negative pressure component with an equation of state like $p=w \rho$ with $-1<w<-0.5$ contributing about $\Omega_{V} \approx 0.65$. There is some leeway in the $(p / \rho)$ of the second component but it is certain that $p$ is negative and $(p / \rho)$ is of order unity (for recent reviews, see [1]). The cosmological constant will provide $w=-1$ while several other candidates based on scalar fields with potentials [2] will provide different values for $w$ in the acceptable range. By and large, component (b) is noticed only in the large scale expansion and it does not cluster gravitationally to a significant extent.

Neither of the components (a) and (b) has laboratory evidence for its existence directly or indirectly. In this sense, cosmology requires invoking the tooth fairy twice to explain the current observations. It would be nice if a candidate could be found which can explain the observations at both small and large scales (so that the tooth fairy needs to be invoked only once). The standard cold dark matter model of the 1980's belongs to this class but - unfortunately - cannot explain the observations. It is obvious from the description in the first paragraph, that any such (single) candidate must have the capacity of leading to different equations of state at different scales and making a transition from $p=0$ at small scales to $p=-\rho$ (say) at large scales. Normal particles (that is, one-particle-excitations of standard quantum field theory) such as weakly interacting massive particles (WIMPs) will usually lead to the equation of state $p=0$ at all scales. On the other hand, homogeneous

*nabhan@iucaa.ernet.in; http://www.iucaa.ernet.in/ paddy field configurations in scalar field models will behave like dark energy with negative pressure and cannot cluster effectively at small scales.

In this paper we examine the possibility of whether a recently proposed [3] candidate - a rolling tachyon arising from string theory - can explain dark matter observations at both small and large scales.

The structure of this scalar field can be understood by a simple analogy from special relativity. A relativistic particle with (one-dimensional) position $q(t)$ and mass $m$ is described by the Lagrangian $L=-m \sqrt{1-\dot{q}^{2}}$. It has the energy $E=m / \sqrt{1-\dot{q}^{2}}$ and momentum $p=m \dot{q} / \sqrt{1-\dot{q}^{2}}$ which are related by $E^{2}=p^{2}+m^{2}$. As is well known, this allows the possibility of having massless particles with finite energy for which $E^{2}=p^{2}$. This is achieved by taking the limit of $m \rightarrow 0$ and $\dot{q} \rightarrow 1$, while keeping the ratio in $E=m / \sqrt{1-\dot{q}^{2}}$ finite. The momentum acquires a life of its own, unconnected with the velocity $\dot{q}$, and the energy is expressed in terms of the momentum (rather than in terms of $\dot{q}$ ) in the Hamiltonian formulation. We can now construct a field theory by upgrading $q(t)$ to a field $\phi$. Relativistic invariance now requires $\phi$ to depend on both space and time $[\phi=\phi(t, \mathbf{x})]$ and $\dot{q}^{2}$ to be replaced by $\partial_{i} \phi \partial^{i} \phi$. It is also possible now to treat the mass parameter $m$ as a function of $\phi$, say, $V(\phi)$ thereby obtaining a field-theoretic Lagrangian $L=-V(\phi) \sqrt{1-\partial^{i} \phi \partial_{i} \phi}$. The Hamiltonian structure of this theory is algebraically very similar to the special relativistic example we started with. In particular, the theory allows solutions in which $V \rightarrow 0, \partial_{i} \phi \partial^{i} \phi \rightarrow 1$ simultaneously, keeping the energy (density) finite. Such solutions will have finite momentum density (analogous to a massless particle with finite momentum $p$ ) and energy density. Since the solutions can now depend on both space and time (unlike the special relativistic example in which $q$ depended only on time), the momentum density can be an arbitrary function of the spatial coordinate. This provides a rich gamut of possibilities in the context of cosmology 顿. 
To examine this scenario in more detail, we will begin with the action which couples such a scalar field to gravity at low energies:

$$
S=\int d^{4} x \sqrt{-g}\left(\frac{R}{16 \pi G}-V(\phi) \sqrt{1-\partial^{i} \phi \partial_{i} \phi}\right),
$$

where $\phi$ is the field and $V(\phi)$ is the potential. Though motivated from string-theoretic considerations, we shall take this action as the starting point and investigate its consequences without worrying about its origin. (In this spirit, we refer to $\phi$ as simply a scalar field, rather than as a tachyonic field). The Einstein equations are

$$
R_{k}^{i}-\frac{1}{2} \delta_{k}^{i} R=8 \pi G T_{k}^{i}
$$

where the stress tensor for the scalar field can be written in a perfect fluid form

$$
T_{k}^{i}=(\rho+p) u^{i} u_{k}-p \delta_{k}^{i}
$$

with

$$
\begin{aligned}
u_{k} & =\frac{\partial_{k} \phi}{\sqrt{\partial^{i} \phi \partial_{i} \phi}} ; u_{k} u^{k}=1 \\
\rho & =\frac{V(\phi)}{\sqrt{1-\partial^{i} \phi \partial_{i} \phi}} \\
p & =-V(\phi) \sqrt{1-\partial^{i} \phi \partial_{i} \phi} .
\end{aligned}
$$

The remarkable feature of this stress tensor is that it could be considered as the sum of two components (a) and (b) described in the first paragraph. To show this explicitly, we break up the density $\rho$ and the pressure $p$ and write them in a more suggestive form as

$$
\rho=\rho_{V}+\rho_{\mathrm{DM}} ; \quad p=p_{V}+p_{\mathrm{DM}}
$$

where

$$
\begin{gathered}
\rho_{\mathrm{DM}}=\frac{V(\phi) \partial^{i} \phi \partial_{i} \phi}{\sqrt{1-\partial^{i} \phi \partial_{i} \phi}} ; \quad p_{\mathrm{DM}}=0 \\
\rho_{V}=V(\phi) \sqrt{1-\partial^{i} \phi \partial_{i} \phi} ;
\end{gathered}
$$

This means that the stress tensor can be thought of as made up of two components - one behaving like a pressure-less fluid, while the other having a negative pressure.

If $V(\phi)$ decreases with $\phi$ and has a minimum at $V=0$ as $\phi \rightarrow \infty$ then it is possible to obtain pressure-less dust solutions by taking the limit $V \rightarrow 0, \partial_{i} \phi \partial^{i} \phi \rightarrow 1$ simultaneously and keeping the energy density finite in the $\rho_{\mathrm{DM}}$ component. If this happens globally at all scales, then - in this limit - the scalar field will behave as pressure-less dust at all scales. In this limit $\rho_{V}$ will vanish. Linear perturbation analysis shows [5] that this component will cluster gravitationally somewhat similar to dust-like particles. In this scenario, the scalar field will merely act as (yet another) candidate for dark matter [5].
(It may be noted that there are still some subtleties related to clustering properties, time-scales etc. which have to be sorted out. But we believe this is indeed possible. For example, some of the problems related to velocities of the condensate particles can be addressed by using solutions which are Lorentz boosted, as explained in [6]).

It is, however, unlikely that such a scenario will be cosmologically acceptable in the absence of another component (b) with negative pressure described in the first paragraph. Unless the clustering property of this scalar field is sufficiently different from that of matter with $p=0$, we will need to still invoke a separate component to describe cosmological observations. More generally, if one assumes that the field $\phi$ has the same configuration at all length scales, then one would end up getting the same density-pressure relation (equation of state) at all scales. However, in the real universe, we know that the dynamics of structure formation and clustering at galactic scales is dominated by the pressure-less fluid component (dark matter), while at large scales, the dynamics of the expansion of the universe is governed by spatially averaged mean density of a pressure-less component and a smooth component with negative pressure. In order to understand these effects, we need to model the scalar field in such a manner that we get different equations of state at different scales. This is possible if we assume that the field $\phi$ has some sort of stochastic behaviour so that its properties at different scales can be obtained by carrying out an averaging over the corresponding scales.

To tackle this complicated issue, we shall define an average of any quantity $A[\phi(t, \mathbf{x})]$ over a length scale $R$, such that the averaged quantity describes the behaviour of the field at that length scale. (This is a fairly standard practice in the study of structure formation; see, for example, chapter 5 of [7].) The average of $A(\phi)$ over a length scale $R$ is defined by smoothing it with a window function $W_{R}$. Mathematically, this is expressed as

$$
\begin{aligned}
A(R) \equiv\langle A(\phi)\rangle_{R} & =\int \frac{d^{3} \mathbf{k}}{(2 \pi)^{3}} A_{\mathbf{k}}(\phi) W_{R}(\mathbf{k}), \\
A_{\mathbf{k}}(\phi) & =\int d^{3} \mathbf{x} A(\phi(\mathbf{x})) e^{i \mathbf{k} \cdot \mathbf{x}},
\end{aligned}
$$

where $W_{R}(\mathbf{k}) \propto \exp \left(-k^{2} R^{2} / 2\right)$ if the window function can be taken to be Gaussian, say. In this case, the behaviour at a scale $R$ will be described by an average potential $\bar{V}_{R}(\bar{\phi})$ obtained by eliminating $R$ between the average of potential $\bar{V}(R)$ and the average of field $\bar{\phi}(R)$ when all the average quantities are obtained using the same window function. In such a description, $\phi$ will sample different parts of $V(\phi)$ at different scales and it is possible to have different equations of state at small and large scales.

To see how it works, consider a simple case in which the field configuration evolves as

$$
\phi(t, \mathbf{x})=A(\mathbf{x}) t+\frac{f(\mathbf{x})}{t^{3}} .
$$


which is a simple generalization of the evolution described in some of the previous works (see, e.g, [3], [8, [9], [10]). When averaged over a length scale $R$ we obtain an effective field

$$
\bar{\phi}(t, R)=A(R) t+\frac{f(R)}{t^{3}}
$$

The dependence of $A(R)$ and $f(R)$ on $R$ will determine the behaviour of the field at different scales. The time dependence of the second term is appropriate if the effective potential at scalar $R$ behaves as

$$
\bar{V}_{R}(\bar{\phi}(t, R))=V_{0}\left(\frac{\phi_{0}}{\bar{\phi}(t, R)}\right)^{2}
$$

which was considered earlier in [8], [11]. For a different potential, the time dependence will be different but in general for $t \gg 1$, the second term will be small compared to the first. [For example, if the potential has the form $\bar{V}_{R}(\bar{\phi}) \propto \exp \left(-\bar{\phi} / \phi_{0}\right)$, the appropriate form of the second term would be $f(R) \exp (-2 t)]$. We shall now show that for a particular choice of $A(R)$, we shall be able to produce expected behaviour of the equation of state at large as well as galactic scales.

At small scales, evolution could have proceeded to the asymptotic limit so that $V \rightarrow 0, \partial_{i} \phi \partial^{i} \phi \rightarrow 1$ and a dust like component prevails, which would require $A(R) \rightarrow 1$. Then we get for the average field

$$
\begin{aligned}
\sqrt{1-\partial^{i} \phi \partial_{i} \phi} & \approx \sqrt{1-\dot{\phi}^{2}-\partial^{\mu} \phi \partial_{\mu} \phi} \\
& =\frac{\sqrt{6 f(R)}}{t^{2}}+\mathcal{O}\left(\frac{1}{t^{4}}\right) .
\end{aligned}
$$

Thus, at these scales, in the limit $t \rightarrow \infty$, we have

$$
\rho_{\mathrm{DM}} \approx \frac{V_{0} \phi_{0}^{2}}{\sqrt{6 f(R)}} ; \quad \rho_{V} \approx 0
$$

This means that the dynamics at galactic scales is dominated by the pressure-less component, whose the energy density is independent of time [3, 5]. This resembles the non-interacting dark matter, which can cluster and is crucial for structure formation in the universe. The time dependence of the second term in the right hand side of (8) was chosen so as to make the energy density $\rho_{\mathrm{DM}}$ independent of time. In a more general scenario, this energy density will be time dependent and will represent the standard growth of structure in the dust-like component in an expanding universe.

Let us now turn into large scales to study the expansion of the universe. Since the fluctuations are likely to decrease with the averaging scales, $\phi(R)$ will be a decreasing function of $R$ and we expect $A(R)$ to have a value less than unity at large scales. Taking $\dot{\phi}(R)=$ $A(R)=$ constant, and $V=V_{0} \phi_{0}^{2} / A(R)^{2} t^{2}$ one can find consistent set of solutions for an $\Omega=1$ FRW model with a power law expansion $a(t) \propto t^{n}$, where (see [8] for a description of this solution):

$$
\phi(t)=\sqrt{\frac{2}{3 n}} t+b_{0} ; \quad V(t)=\frac{3 n^{2}}{8 \pi G} \sqrt{1-\frac{2}{3 n}} \frac{1}{t^{2}}
$$

with $b_{0}$ being some constant. Our model reproduces the correct behaviour expected at large scales, provided we identify

$$
A(R)=\sqrt{\frac{2}{3 n}}, \quad V_{0} \phi_{0}^{2}=\frac{n}{4 \pi G} \sqrt{1-\frac{2}{3 n}} .
$$

Thus the average value of $\phi$ being different at different scales allows the possibility of the same scalar field exhibiting different equations of state at different scales. The rate of expansion of the universe is essentially determined by $A(R)$ at the larger scales.

Since the same physical entity provides the dark matter at all scales in this scenario, one certainly expects a relation between the energy densities contributed by dark matter $\left(\Omega_{m}\right)$ and dark energy $\left(\Omega_{V}\right)$. In our model, the energy densities for the two components are given by

$$
\begin{aligned}
\rho_{\mathrm{DM}} & \approx \frac{V_{0} \phi_{0}^{2}}{\sqrt{1-A(R)^{2}} \frac{1}{t^{2}}}=\frac{n}{4 \pi G} \frac{1}{t^{2}}, \\
\rho_{V} & \approx \frac{V_{0} \phi_{0}^{2} \sqrt{1-A(R)^{2}}}{A(R)^{2} t^{2}}=\frac{3 n^{2}}{8 \pi G}\left(1-\frac{2}{3 n}\right) \frac{1}{t^{2}} .
\end{aligned}
$$

(It may be necessary to choose the value of $V_{0} \phi_{0}^{2}$ in a particular range to match the values of the energy densities we observe today. This could be considered a fine tuning of the parameters, which we need to resort to at this stage in the absence of a more fundamental understanding of the scalar field. It is no worse or better than the fine tuning which is required in any other model for dark energy.) However, the ratio of the energy densities $\rho_{V} / \rho_{\mathrm{DM}}$ is independent of time, and is related to the mean value of the scalar field at large scales by

$$
\frac{\rho_{V}}{\rho_{\mathrm{DM}}}=\frac{1}{A(R)^{2}}-1 \text {. }
$$

In fact, a similar equation holds for the ratio of the two components at all scales. As one proceeds from smaller to larger scales, the dark matter contribution decreases and the dark energy contribution increases.

This result can be converted into a clear prediction for cosmology by expressing the above equation in terms of the rate of expansion $n$ :

$$
n=\frac{2}{3}\left(1+\frac{\rho_{V}}{\rho_{\mathrm{DM}}}\right) .
$$

For the values accepted at present $\rho_{V} / \rho_{\mathrm{DM}} \approx 2$, we get $a(t) \propto t^{2}$. Such a rate of growth is consistent with supernova observations. (The age of the universe in any accelerating model [with $\Omega_{t o t}=1, a(t) \propto t^{n}, n>1$ ] will be $t_{0} \approx n / H_{0}$, which is higher than the conventional models with $t_{0} \approx 1 / H_{0}$. Any model which agrees with the 
SN observations and has entered an accelerating phase in the recent past will have this feature and our model with $n \approx 2$ is no different.) This relation between (i) the amounts of dark matter and dark energy present in the universe and (ii) the expansion rate is potentially testable by observations. It may be stressed that in our model, the evolution of the single scalar field governs the time dependence of both $\rho_{D M}$ and $\rho_{V}$. This is equivalent to saying that there is interaction and energy exchange between the two components and the energy is not conserved locally for the dark matter and dark energy components separately (which would imply $\rho_{\mathrm{DM}} \propto a^{-3}$ and $\rho_{V}=$ constant).

Incidentally, it may be possible to put constraints on $n$ from CMB observations as well. The pressure term in the linear perturbation equation in this model has a factor $\left(1-\dot{\phi}^{2}\right) k^{2}$ where $k$ is the wave number [5]. For the solution (13), this factor is $[1-(2 / 3 n)] k^{2}$ and the standard results can be used with a rescaling of $k$. But since the angular scales of features in CMB anisotropy depends on this rescaling, it will lead to an $n$ dependent rescaling of Doppler peaks etc. 12. Hence, CMB observations can provide another constraint on $n$.

The really serious test of the model will arise from the non-linear small scale dynamics of the clustering and galaxy formation scenarios. This is a hard problem which we have not studied in this paper; instead we have introduced an ansatz for the form of scalar field at different scales by hand. It is necessary to investigate this model further and show that the basic ansatz is correct and the details do not run into any contradiction. While this remains to be done, we consider it very attractive that the single entity can possibly exhibit different equations of state at different scales in the universe. Such a scenario has nuances (for example, for CMB observations [12]) which have not been explored in conventional cosmology before.

We thank Ashoke Sen for useful discussions. T.R.C. is supported by the University Grants Commission, India.
[1] S. Sarkar, in EPS International Conference on High Energy Physics, Budapest, 2001 (D. Horvath, P. Levai, A. Patkos, eds.), JHEP Proceedings Section, PrHEPhep2001/299; B. Leibundgut, Ann. Rev. Astron. \& Astrophys. 39, 67 (2001).

[2] B. Ratra, and P.J.E. Peebles, Phys. Rev. D 37, 3406 (1988); C. Wetterich, Nuclear Physics B 302, 668 (1988); P.G. Ferreira and M. Joyce, Phys. Rev. D 58, 023503 (1998); J. Frieman, C.T. Hill, A. Stebbins, and I. Waga, (1995) Phys. Rev. Lett. 75, 2077; P. Brax and J. Martin Phys. Lett. B 468, 40 (1999); P. Brax and J. Martin Phys. Rev. D 61, 103502 (2000); V. Sahni and A. A. Starobinsky, IJMP 9, 373 (2000); L.A. Ureña-López and T. Matos, Phys. Rev. D 62, 081302 (2000); T. Barreiro, E.J. Copeland and N.J. Nunes Phys. Rev. D 61, 127301 (2000); I. Zlatev, L, Wang and P.J. Steinhardt Phys. Rev. Lett. 82, 896 (1999); A. Albrecht and C. Skordis Phys. Rev. Lett. 84, 2076 (2000); N. Bilic, G. B. Tupper and R. D. Viollier, Phys. Lett. B 535, 17 (2002).

[3] A. Sen, arXiv: hep-th/0204143; arXiv: hep-th/0203211; arXiv: hep-th/0203265 and references cited therein.

[4] This interpretation may be of some historic/pedagogical value. Generalizing the non-relativistic particle Lagrangian $L_{N R}=(1 / 2) \dot{q}^{2}-V(q)$ by changing $q(t)$ to field $\phi(t, \mathbf{x})$ will lead to standard scalar field theory with a potential $V(\phi)$, while generalizing the relativistic particle Lagrangian leads to the theory we are studying in the paper. Historically, one proceeded from nonrelativistic classical mechanics to nonrelativistic quantum mechanics and attempted to generalize the Schrodinger wave equa- tion to relativistic wave equations. Instead, if one had proceeded from nonrelativistic classical mechanics to relativistic classical mechanics and upgraded the $q$ to a field, one would have naturally led to this Lagrangian. We do not know whether such an attempt was ever made in the early days of quantum field theory. This gives another motivation to study such a scalar field independent of its string-theoretic origin.

[5] T. Padmanabhan, unpublished; A. Frolov, L. Kofman and A. Starobinsky, arXiv:hep-th/0204187; G. Shiu and I. Wasserman, arXiv:hep-th/0205003.

[6] A.Sen, hep-th/0204143 [see the discussion after equa$\operatorname{tion}(25)]$

[7] T. Padmanabhan, 1993, Structure formation in the universe (Cambridge University Press).

[8] T. Padmanabhan, arXiv:hep-th/0204150.

[9] G.W. Gibbons, arXiv:hep-th/0204008; M. Fairbairn and M.H. Tytgat, arXiv:hep-th/0204070; S. Mukohyama, arXiv:hep-th/0204084. D. Choudhurv. D. Ghoshal, D. P. Jatkar and S. Panda, arXiv:hep-th/0204204.

[10] C. Acatrinei and C. Sochichiu, arXiv:hep-th/0104263; S. H. Alexander, Phys. Rev. D 65, 023507 (2002) arXiv:hep-th/0105032; A. Mazumdar, S. Panda and A. Perez-Lorenzana, Nucl. Phys. B 614, 101 (2001) arXiv:hep-ph/0107058; S. Sarangi and S. H. Tye, arXiv:hep-th/0204074.

[11] A. Feinstein, arXiv:hep-th/0204140;

[12] T. Padmanabhan, work in progress. 